\title{
Cyclodextrin-mediated gold nanoparticles as multisensing probe for the selective detection of hydroxychloroquine drug
}

\author{
Jaise Mariya George and Beena Mathew ${ }^{\dagger}$ \\ School of Chemical Sciences, Mahatma Gandhi University, Kottayam-686560, Kerala, India \\ (Received 4 August $2020 \bullet$ Revised 9 November 2020 • Accepted 22 November 2020)
}

\begin{abstract}
Cyclodextrin $(\beta$-CD) modified gold nanoparticles (AuNP) were rapidly synthesized using microwave assisted procedure. Parameters, such as time, $\mathrm{pH}$ and concentrations of $\beta-\mathrm{CD}$ and gold, were optimized for the synthesis of $\beta$-CD-AuNP. The addition of enantiomers and racemic mixture of hydroxychloroquine (R-HCQ, S-HCQ and RS-HCQ) drugs and their interaction with $\beta$-CD led to a red shift in the surface plasmon resonance of $\beta$-CD-AuNP. The changes associated with the introduction of HCQ in $\beta$-CD-AuNP were studied using various characterization techniques such as UV-vis, FT-IR, XRD, dynamic light scattering, zeta potential, transmission electron microscopy, fluorescence spectroscopy and electrochemical techniques. The host-guest interaction of $\beta$-cyclodextrin with S-HCQ, RHCQ and RS-HCQ resulted in the aggregation of gold nanoparticles. The surface plasmon resonance at $521 \mathrm{~nm}$ for $\beta$ CD-AuNP was shifted to 600, 620 and $670 \mathrm{~nm}$ on the addition of S-HCQ, R-HCQ and RS-HCQ, respectively, with a color change from pink to blue. The selectivity and sensitivity of the developed system for RS-HCQ were investigated and the limit of detection ( $\mathrm{LOD}=3 \mathrm{~s} / \mathrm{m}$ ) was found to be $2.61,0.15$, and $0.85 \mathrm{nM}$ for optical, fluorescence and electrochemical methods, respectively. The successful monitoring of RS-HCQ drug in pharmaceutical samples is possible with these techniques.
\end{abstract}

Keywords: Gold Nanoparticles, $\beta$-Cyclodextrin, Hydroxy Chloroquine, Enantiomeric, Sensing

\section{INTRODUCTION}

The integration of nanomaterials in synthesis and analytical instrumentation is a significant area of research that is widely used to monitor different kinds of chemical species in biological and environmental samples [1-3]. Among metal nanoparticles, gold nanoparticles (AuNP) are accepted candidates for colorimetric sensing of various species, including metal ions, drugs, biomolecules and pesticides with simple sample treatment [4-8] due to the unique physicochemical properties of AuNP. The unique optical properties of AuNPs are associated with surface plasmon resonance, which depends upon the size, shape, capping agents, interparticle distance and medium refractive index, which makes them suitable nanoprobe for the sensing of different analytes $[9,10]$. The extent of interaction between AuNP and analytes makes them suitable for colorimetric assays that control dispersion and aggregation of AuNPs. The large molar extinction coefficient and broad energy bandwidth of AuNPs are suitable for the electron/energy transfer between AuNPs and various analyte molecules, which is responsible for AuNPs to act as hyperquencher or turn on sensors in fluorescence sensing [5,11].

Hydroxychloroquine sulfate drug (HCQ) (Fig. 1) is a chiral drug derived from 4-aminoquinoline, which is used for malaria. It is also used for rheumatoid arthritis, lupus erythematosus, and inflammatory and skin diseases [12-16]. Hydroxychloroquine is considered a

\footnotetext{
${ }^{\dagger}$ To whom correspondence should be addressed.

E-mail: beenamathew@mgu.ac.in, beenamscs@gmail.com

Copyright by The Korean Institute of Chemical Engineers.
}

valid treatment option for pandemic COVID-19 outbreak [17]. After intake, HCQ is quickly absorbed in the intestine and accumulated in the liver, lungs, spleen and kidneys. Partial conversion of the active metabolite occurs in the liver and is excreted through the kidneys [18]. The toxicity of the HCQ in ophthalmic concern arises by the deposition of drug in all tissues containing melanin and develop changes in choroid, iris and importantly in the retinal pigment epithelium, thereby producing retinopathy [14-16]. Therefore, the pharmaceutical development of HCQ needs easy, fast and accurate analytical techniques for the determination and quantification. The individual enantiomeric properties of HCQ against arthritis and malaria are unknown; somehow it has been estimated that the S-HCQ exhibits higher antimalarial property than R-HCQ in rats [19].<smiles>CCN(CCO)CCCC(C)Nc1ccnc2cc(Cl)ccc12</smiles>

Fig. 1. Chemical structure of hydroxychloroquine ((RS)-2-[\{4-[(7chloroquinolin-4-yl)amino]pentyl\}(ethyl)amino]ethanol). 
The traditional methods for the determination of HCQ are nonaqueous titrimetric [20] or spectrophotometric methods [21]. Due to the non-specificity of these methods, a number of HPLC methods were developed for the determination of HCQ in biological fluids, such as spectrophotometric [19], fluorescence [22], isocratic HPLC system with UV detector [23] and tandem mass spectrometry [24]. Some of these methods may have some disadvantages [25]. Electrochemical methods such as differential pulse voltammetry [26], square-wave voltammetry [25] and potentiometric techniques [27] have also been used for the sensing of HCQ. Due to the synergistic effect between the metal nanoparticles and $\beta-\mathrm{CD}$, they have been exploited for the selective detection of HCQ. This sort of sensor is simple, low cost and produces a reliable response over a wide concentration range with low limit of detection owing to the physico-chemical properties of AuNP and $\beta$-CD.

Cyclodextrins are naturally occurring cyclic oligosaccharides linked by $\alpha$-1,4-glycosidic linkages of six, seven, or eight glucose units [28]. Among these non-toxic green cyclodextrins, $\beta$-CD significantly forms highly selective inclusion guest-host complexes with strong binding constants [29]. The less hydrophilic internal cavity and the hydrophilic exterior of $\beta$-CD enable it to make supramolecular interactions such as electrostatic, hydrogen bonding, van der Waals forces and hydrophobic interactions with organic, inorganic and bio molecules [30]. The inner cavity of cyclodextrins can completely or partly enclose the guest molecules, depending upon its size and dimensions of the macrocyclic structure [31]. Cyclodextrins are widely used in chromatography and capillary electrophoresis as chiral selector attributed to its inclusion effect [32], which is effectively needful in pharmaceutical industries for enantiomerically pure drugs [33].

Detailed studies of colorimetric and fluorescence-based detection methods of HCQ are not much available and the interaction of HCQ with a pharmaceutical excipient such as cyclodextrin is also an interesting area of research. In this context, the main objective of this manuscript is the cyclodextrin mediated synthesis of gold nanoparticles via microwave assisted method, exploitation of the intrinsic chirality of $\beta$-CD for the sensing of enantiomeric hydroxychloroquine drugs and further evaluation of its individual enantiomer with the aid of $\beta$-CD-AuNP. The colorimetric changes induced by the presence of drug in $\beta$-CD-AuNP and extended application in optical, fluorescence and electrochemical techniques are investigated in this study.

\section{MATERIALS AND METHODS}

Auric chloride and $\beta$-CD were obtained from Sigma Aldrich. R\&S hydroxychloroquine sulfate drug was obtained from Microtherapeutic Research Lab, Chennai, India as kind gift samples. All chemicals used were of analytical grade and without any extra purification. Millipore $^{\mathbb{R}}$ water was used throughout the experiments.

\section{Instrumentation}

The formation of $\beta$-CD-AuNP and its sensing properties toward HCQ drug was studied using UV-vis absorption spectrophotometer (Shimadzu UV-2450, Japan). The functional group interaction was predicted using FT-IR spectroscopy by Perkin Elmer-400, (Singapore) spectrometer with ATR attachment. XRD pattern of studied materials was recorded using PANalytic X'PERT-PRO Xray spectrometer. Morphological studies were investigated using JEOL JEM-2010 transmission electron microscopy. The electrochemical work, including cyclic voltammetry and differential pulse voltammetry, was carried out with Biologic SP-200 (Germany) electrochemical workstation. DLS and zeta potential measurements were carried out by (Horiba SZ-100, Japan) scientific nanoparticle analyzer. Gold electrode modified with $\beta$-CD-AuNP, a platinum wire and $\mathrm{Ag} / \mathrm{AgCl}$ were used as working electrode, auxiliary electrode and a reference electrode, respectively. All electrochemical experiments were performed in $0.1 \mathrm{M}$ PBS at room temperature.

\section{Experimental}

\section{2-1. Microwave Synthesis of $\beta$-CD-AuNP}

In a typical synthesis, about $12 \mathrm{~mL}$ of water was taken along with $0.01 \mathrm{M} \mathrm{HAuCl}_{4}$ and $4 \mathrm{~mL}$ of $0.01 \mathrm{M} \beta$-CD solution. $\mathrm{pH}$ of this solution was adjusted by adding $1 \mathrm{M} \mathrm{NaOH}$. This solution was kept in a domestic microwave oven $(800 \mathrm{~W})$ with a frequency of $2,450 \mathrm{MHz}$ and irradiated for 1 minute. The colorless solution turned to pink color solution after the microwave irradiation, indicating the formation of gold nanoparticles. The UV-vis absorption spectra for each experiment were monitored for confirming the formation of $\beta$-CD-AuNP. The optimized conditions for the formation of AuNP were monitored using UV-vis spectroscopy. The colloidal solution of AuNP was cooled to room temperature and kept at $4{ }^{\circ} \mathrm{C}$ for further experimental study.

2-2. Optical Sensing of RS-HCQ Using $\beta$-CD-AuNP

To study the colorimetric detection of RS-HCQ, $2 \mathrm{~mL}$ of the $\beta$ CD-AuNP was mixed with $50 \mu \mathrm{L}$ of $1 \mathrm{mM}$ HCQ solution without any change in $\mathrm{pH}$ at room temperature. This solution was shaken for 1 minute and kept standing for a few minutes. As a result, the pink colored colloidal solution of $\beta$-CD-AuNP changed into dark blue color solution due to the interaction between drug and nanoparticles. The resultant changes in surface plasmon resonance absorption were observed using UV-vis spectrophotometer. In a similar way R-HCQ and S-HCQ solutions $(0.01 \mu \mathrm{M})$ were added to $\beta$ $\mathrm{CD}$-AuNP solution and corresponding changes were monitored.

2-3. Fluorescence Response of RS-HCQ Using $\beta$-CD-AuNP

For fluorescence sensing, the excitation wavelength selected was $512 \mathrm{~nm}$. The emission wavelength was recorded from 650 to 850 $\mathrm{nm}$ with a slit width of $5 \mathrm{~nm}$. To study the fluorescence response of the RS-HCQ with $\beta$-CD-AuNP, $3 \mathrm{ml}$ of the synthesized colloidal solution of $\beta$-CD-AuNP was mixed with concentrations of $\mathrm{R}$ HCQ, S-HCQ and RS-HCQ solution ranging from 0.01 to 0.06 $\mu \mathrm{M}$. The solutions were mixed well and fluorescence spectra were monitored. Selectivity and interference studies were also carried out to determine the sensitivity of the proposed sensor.

$2-4$. Electrochemical Sensing of RS-HCQ using $\beta$-CD-AuNP Modified $\mathrm{AuE}$

A systematic cleaning of the electrode is necessary for electrode fabrication. The mechanical polishing of AuE used alumina slurry and diamond slurry. The electrode surface was washed with ultrapure water several times to remove the adhered unwanted particles to obtain a mirror finish polished surface. Again, AuE was sonicated for several minutes in an ethanol water mixture $(1: 1)$. After thorough washing, a small aliquot of $\beta$-CD-AuNP colloidal solution was cast on the surface of the cleaned $\mathrm{AuE}$ and dried in 
air. After complete drying, the obtained $\beta$-CD-AuNP/AuE was instantly used for electrochemical experiments, which included cyclic voltammetry (CV) and differential pulse voltammetric (DPV) methods. A stock solution of $0.05 \mu \mathrm{M}$ of RS-HCQ was prepared by dissolving the required amount in ultrapure water. Required amount of RS-HCQ stock solution was added to the electrochemical cell containing $0.1 \mathrm{M}$ PBS as the electrolyte. Cyclic voltammograms were recorded using electrochemical workstation by sweeping potential from 0.6 to $1.3 \mathrm{~V}$ at a scan rate of $100 \mathrm{mV} / \mathrm{s}$. The electrode was optimized under several conditions with respect to $\mathrm{Ag} / \mathrm{AgCl}$ electrode at room temperature.

\section{RESULTS AND DISCUSSION}

\section{Optimization of Synthesis of $\beta$-CD-AuNP}

UV-vis absorption spectroscopy is extensively used to ascertain the formation, size and stability of metal nanoparticles in aqueous colloidal solutions. Due to surface plasmon resonance (SPR), the position of the peak maximum provides information about the size and morphology of gold nanoparticles [34]. The primary confirmation of nanoparticle formation is attainable from the visual color change from to pink in the case of $\beta$-CD-AuNP. $\beta$-CD was colorless and no absorption peak was observed in the UV-vis spectrum (Fig. 2). The addition of auric chloride solution and $\mathrm{pH}$ change did not induce any color change in the system at room temperature. During microwave irradiation, the color of the reaction mixture changed to pink solution. Fig. 2 represents the UV-vis spectra of the reaction medium at $30 \mathrm{~s}$ intervals of time during the synthesis of $\beta$-CD-AuNP. After $30 \mathrm{~s}$ of microwave irradiation, an SPR band appeared at $521 \mathrm{~nm}$ and its intensity further increased with time without altering the absorption maxima. Thus, the microwave mediated synthesis of $\beta$-CD-AuNP was completed within $60 \mathrm{~s}$. The primary $\mathrm{OH}$ groups present in the $\beta$-CD acted as the reducing agent, which reduced $\mathrm{Au}^{3+}$ ions and oxidized to carboxylic acid. The interactions of these carboxylic acid groups provided the stability to nanoparticles and prevented further aggregation of nanoparticles [35].

For the synthesis of $\beta$-CD-AuNP in one minute, the effect of concentration of $\beta$-CD was studied by changing the concentration of $\beta$-CD from (0.001-0.015 M) and the corresponding change

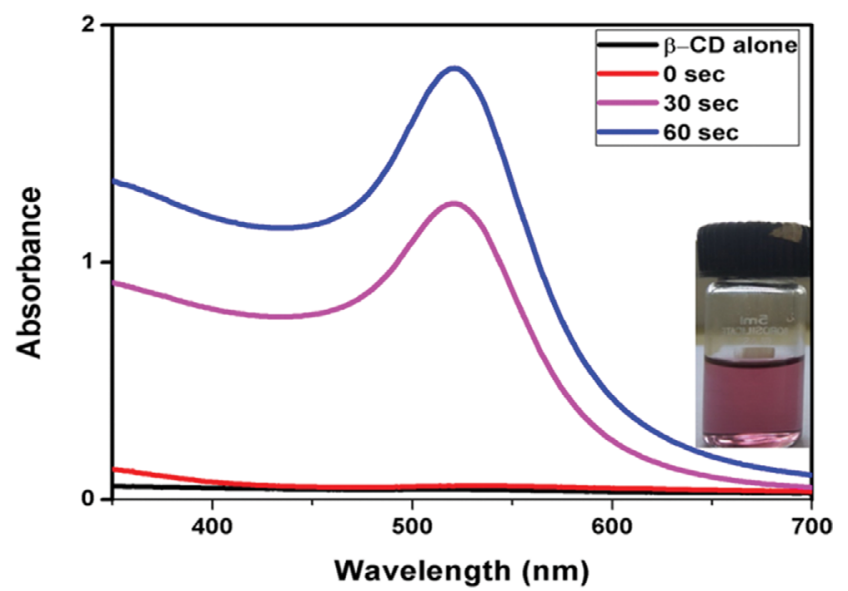

Fig. 2. UV-vis spectra of the formation of $\beta$-CD-AuNP at different intervals of time.

in SPR was monitored (Fig. 3(a)). As the concentration of $\beta-\mathrm{CD}$ increased, the peak intensity increased and reached maximum for $0.01 \mathrm{M}$ concentration, suggesting the effective stabilization provided by $\beta$-CD to prevent agglomeration, resulting in $\beta$-CD-AuNP with almost uniform size. Above $0.01 \mathrm{M}$ concentration of $\beta$-CD, the peak intensity decreased due to agglomeration of nanoparticles.

Effect of $\mathrm{pH}$ played a vital role in the controlled synthesis and formation of AuNP. The favorable $\mathrm{pH}$ for the reduction of metallic precursors using $\beta$-CD for uniform nanoparticles was around $10-11$, as mentioned in previous reports and higher $\mathrm{pH}$ resulting aggregation of nanoparticles [37]. The $\mathrm{pH}$ of the reaction system was varied by adding $1 \mathrm{M} \mathrm{NaOH}$. In Fig. $3(\mathrm{~b})$ it is clear that at $\mathrm{pH}$ 10.0 a sharp, intense peak was obtained with a high extinction coefficient. Above $\mathrm{pH} 10.0$, the peak intensity decreased and also the stability of the nanoparticles was reduced. Therefore, $\mathrm{pH} 10$ was selected as suitable $\mathrm{pH}$ for $\beta$-CD-AuNP synthesis.

Fig. 3(c) illustrates the effect of amount of $\mathrm{HAuCl}_{4}$ to augment the formation AuNP. 0.01 $\mathrm{M} \mathrm{HAuCl}_{4}$ was selected during the course of the experiment and the amount of $\mathrm{HAuCl}_{4}$ varied from 500 to $800 \mu \mathrm{L}$ (Fig. 2(c)). As the amount of $\mathrm{HAuCl}_{4}$ increased, the peak intensity also increased due to availability of the $\mathrm{Au}^{3+}$ enhanced. Thus, for the synthesis of AuNP, $800 \mu \mathrm{L}$ of $0.01 \mathrm{M} \mathrm{HAuCl}_{4}$ was
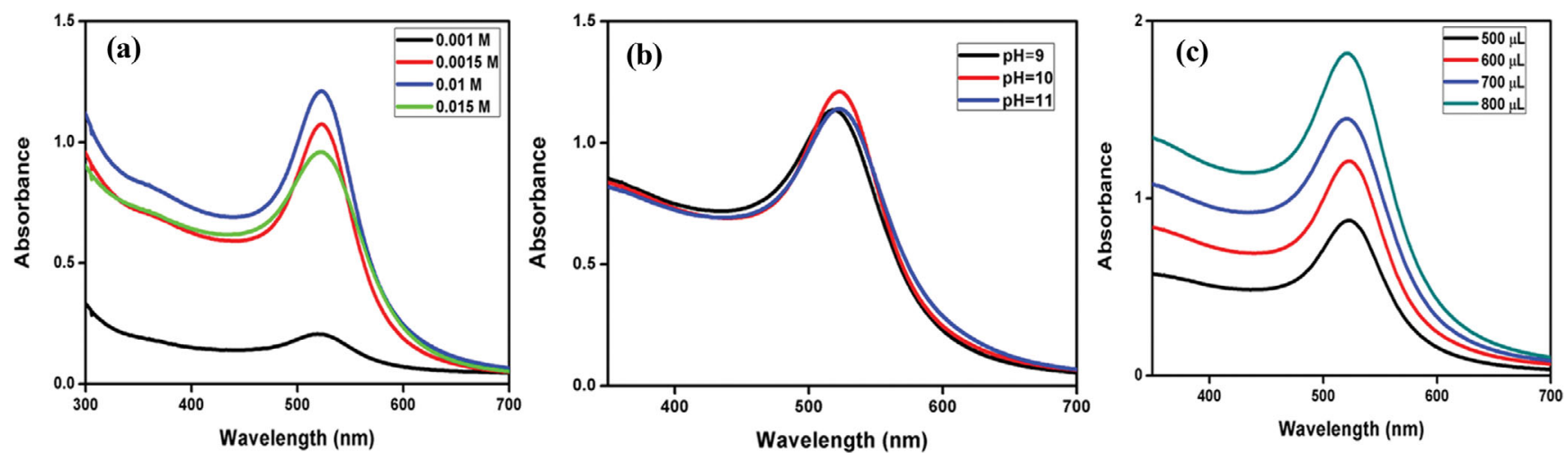

Fig. 3. UV-vis. spectra of (a) $\beta$-CD-AuNP prepared at different $\beta$-CD concentrations $(0.001-0.015 \mathrm{~mol} / \mathrm{L})$, (b) dependence of different $\mathrm{pH}$ on the formation of $\beta$-CD-AuNP and (c) effect of different amount of $0.01 \mathrm{M} \mathrm{HAuCl}_{4}$ in the formation of $\beta$-CD-AuNP. 


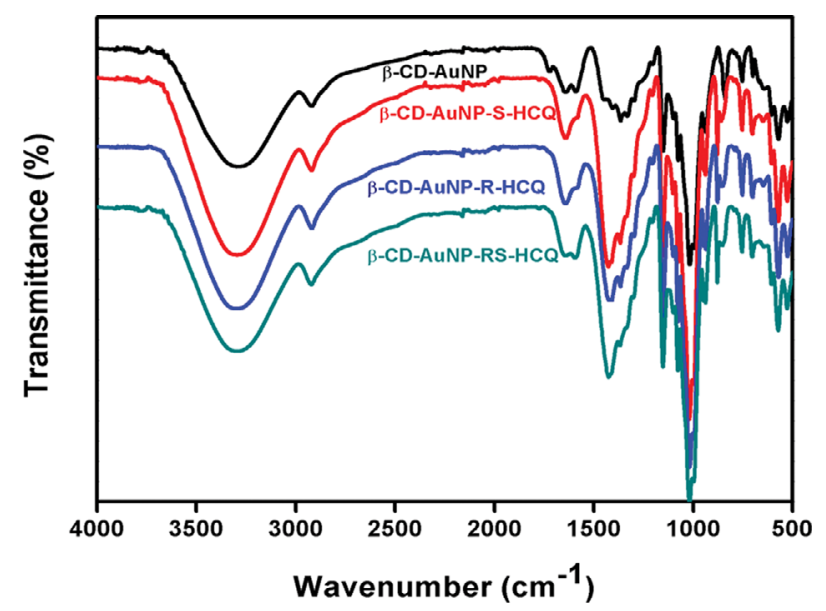

Fig. 4. FTIR spectra of $\beta$-CD-AuNP, $\beta$-CD-AuNP-S-HCQ $\beta$-CDAuNP-R-HCQ, and $\beta$-CD-AuNP-RS-HCQ.

selected as the suitable amount for the controlled synthesis of $\beta$ CD-AuNP.

\section{Characterization}

2-1. FTIR Analysis

The FTIR spectra revealed the functional groups involved in the formation of $\beta$-CD-AuNP and its interaction with hydroxychloroquine (Fig. 4). The band at $3,283 \mathrm{~cm}^{-1}$ in the $\beta$-CD-AuNP shifted to $3,295 \mathrm{~cm}^{-1}$ in $\beta$-CD-AuNP-RS-HCQ and also corresponding changes into other single enantiomers. The shift in the $\mathrm{O}-\mathrm{H}$ stretching frequency indicated the hydrogen bond interaction between the $\beta$-CD-AuNP and hydroxy chloroquine, which resulted in the color change of the colloidal $\beta$-CD-AuNP. The peak at $2,920 \mathrm{~cm}^{-1}$ indicated the $\mathrm{C}-\mathrm{H}$ stretching frequency of $\beta-\mathrm{CD}$, which

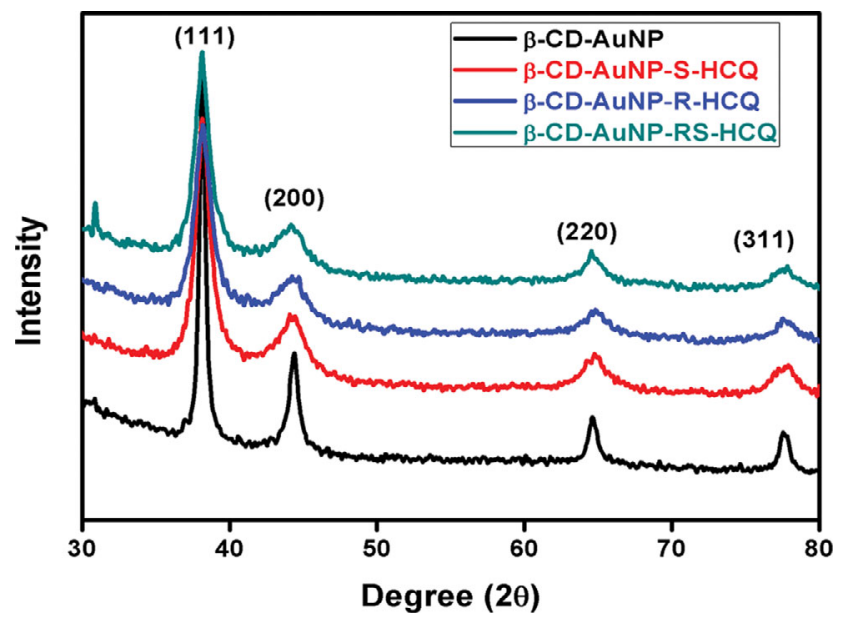

Fig. 5. XRD pattern of $\beta$-CD-AuNP, $\beta$-CD-AuNP-S-HCQ, $\beta$-CDAuNP-R-HCQ, and $\beta$-CD-AuNP-RS-HCQ.

is more or less unaffected with drug molecules. The H-O-H bending frequency observed at $1,644 \mathrm{~cm}^{-1}$ in $\beta$-CD-AuNP was weak, became more intense in the presence of hydroxy chloroquine, which is attributed to the major involvement of hydrogen bonding in sensing. The peak around $1,426 \mathrm{~cm}^{-1}$ corresponds to $\mathrm{C}=\mathrm{C}$ aromatic stretching frequency, which is absent in $\beta$-CD-AuNP is markedly shifted in $\beta$-CD-AuNP-RS-HCQ.

\section{2-2. XRD Analysis}

The indexed XRD pattern for $\beta$-CD-AuNP portrayed in Fig. 5 gives information about the crystalline nature of the synthesized nanoparticles. For gold nanoparticles, the characteristic four distinct Bragg peaks appeared at $38.21^{\circ}, 44.41^{\circ}, 64.63^{\circ}$ and $77.55^{\circ}$.

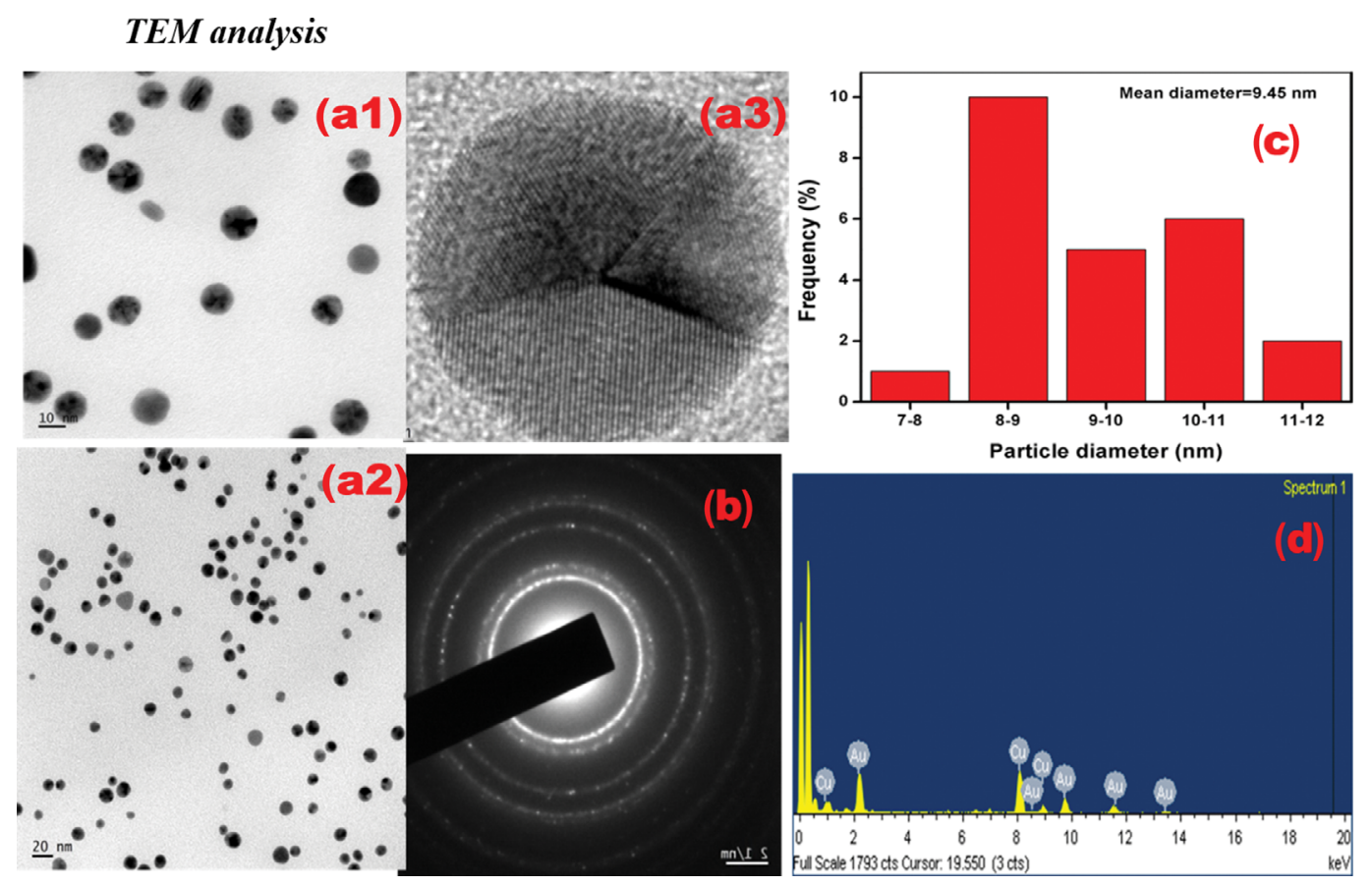

Fig. 6. TEM images of (a1)-(a3) $\beta$-CD-AuNP under different magnifications, (b) SAED pattern of $\beta$-CD-AuNP, (c) particle size histogram of $\beta$-CD-AuNP, and (d) EDX spectra of $\beta$-CD-AuNP. 
These are assigned to the reflections from the crystal planes of (111), (200), (220) and (311), which is in agreement with the JCPDS 00001-1172 [36] and indicates the face-centred cubic structure of synthesized nanoparticles. The more intense Bragg reflections from (111) plane compare than three (200), (220), and (300) planes, suggesting that the preferential growth of nanoparticles is along the direction of (111) plane. HCQ incorporated $\beta$-CD-AuNP shows similar diffraction patterns with that of AuNP with reduced intensity, suggesting the strong interaction between the $\beta$-CD-AuNP with HCQ. This interaction is higher for $\beta$-CD-AuNP-R-HCQ than with $\beta$-CD-AuNP-S-HCQ. This demonstrates the reduction in the corresponding peak intensity, which supports the selective interaction of $\beta$-CD-AuNP with enantiomeric sensing of HCQ. The broad diffraction patterns attained for the HCQ incorporated $\beta$-CD-AuNP due to losing of crystallinity due to the aggregation of nanoparticles resulting from the inclusion complexes among $\beta$ CD and HCQ [37].

Morphological studies of $\beta$-CD-AuNP and its interaction with different kinds of HCQ were carried out using TEM analysis. Fig. $6(\mathrm{a} 1)$, (a2) and (a3) are the TEM images of $\beta$-CD-AuNP at different magnifications. Most of the synthesized gold nanoparticles are spherical and monodispersed with an average particle size of 9.45 $\mathrm{nm}$ obtained from particle size histogram (Fig. 6(c)). Clear and highly ordered lattice fringes are observed in the HR-TEM images of $\beta$-CD-AuNP. Fig. 6(d) portrays bright circular ring-like patterns in the selected area electron diffraction (SAED) indicated pure crystalline nature ( $\mathrm{fcc}$ ) of $\beta$-CD-AuNP. The ring patterns are indexed according to (111), (200), (220), and (311) planes of fcc crystalline structure of gold, which supports the XRD results. The TEM images obtained after the addition HCQ into the $\beta$-CD-AuNP are depicted in Fig. 7. With the addition of S-HCQ into $\beta$-CD AuNP, the average particle size increased and interparticle distances among the AuNP were reduced, resulting in the aggregation of nanoparticles. Further enhancement of aggregation is observed in the case of $\beta$ CD-AuNP-R-HCQ. The coulombic interaction between RS-HCQ and $\beta$-CD-AuNP is highly extended and the random aggregation of nanoparticles results, which is obviously clear in Fig. 7(c).

2-3. Energy Dispersive X-ray (EDAX) Analysis

The elemental composition of $\beta$-CD-AuNP and effect of binding of HCQs were studied by means of energy dispersive X-ray analysis (EDAX). The EDAX spectrum of $\beta$-CD-AuNP exhibited signals at 2.31, 8.52, 9.60 and $11.50 \mathrm{keV}$ (Fig. S1) [38]. Compared
Table 1. DLS and zeta potential values of $\beta$-CD-AuNP, $\beta$-CD-AuNPSHCQ $\beta$-CD-AuNP-RHCQ and $\beta$-CD-AuNP-RS-HCQ

\begin{tabular}{lcc}
\hline \hline \multicolumn{1}{c}{ Sample } & $\begin{array}{c}\text { Zeta potential } \\
(\mathrm{mV})\end{array}$ & $\begin{array}{c}\text { DLS values } \\
(\mathrm{nm})\end{array}$ \\
\hline$\beta$-CD-AuNP & -22.7 & 26.6 \\
$\beta$-CD-AuNP-S-HCQ & -23.4 & 54.0 \\
$\beta$-CD-AuNP-R-HCQ & -25.3 & 92.7 \\
$\beta$-CD-AuNP-RS-HCQ & -27.7 & 161.5 \\
\hline
\end{tabular}

with $\beta$-CD-AuNP, $\beta$-CD-AuNP-S-HCQ has a higher weight percentage of gold due to the agglomeration with S-HCQ as depicted in Fig. $\mathrm{S}(1 \mathrm{a})$. As the agglomeration increased from $\beta$-CD-AuNP$\mathrm{R}-\mathrm{HCQ}$ to $\beta$-CD-AuNP-RS-HCQ, the weight percentage of $\mathrm{Au}$ increased (Fig. S(1b) \& (c)). The signals of copper are from the copper grid used for the sample preparation.

2-4. DLS and Zeta Potential Measurements

The dynamic light scattering (DLS) technique and zeta size measurements were carried out to obtain the hydrodynamic diameter and surface charge, respectively, associated with this system. As seen in Fig. $\mathrm{S}(2 \mathrm{a})$, the particle size of $\beta$-CD-AuNP is found to be 26.6 $\mathrm{nm}$, which is usually higher than the values of TEM measurements because of the hydrodynamic diameter obtained from DLS technique. The variation in zeta potential and DLS values from $\beta$-CDAuNP, $\beta$-CD-AuNP-SHCQ, $\beta$-CD-AuNP-RHCQ to $\beta$-CD-AuNPRS-HCQ are compiled in Table 1 . The particle size measurements by dynamic light scattering of $\beta$-CD-AuNP-SHCQ, $\beta$-CD-AuNPRHCQ to $\beta$-CD-AuNP-RS-HCQ are displayed in Fig. S2. After the addition of HCQ into the $\beta$-CD-AuNP, the zeta potential value became more negative, which points out the binding of HCQ onto the surface and the zeta potential difference in each case associated with the variation in electric dipole on each molecule.

3. Mechanistic Study of $\beta$-CD-AuNPs for the Colorimetric Sensing of RS-HCQ

The estimation of minimum detectable concentration of $\mathrm{S}, \mathrm{R}$ and RS-HCQ was obtained by adding different concentrations of drugs into $\beta$-CD-AuNP aqueous solutions and the color changes associated with the aggregation of nanoparticles were monitored through UV-vis. spectroscopy. As the concentration of target analyte concentrations increased, the $\beta$-CD-AuNP solution gradually turned blue, proposing an increased aggregation of nanoparticles. Consequently, the characteristic SPR peak at $521 \mathrm{~nm}$ was gradu-
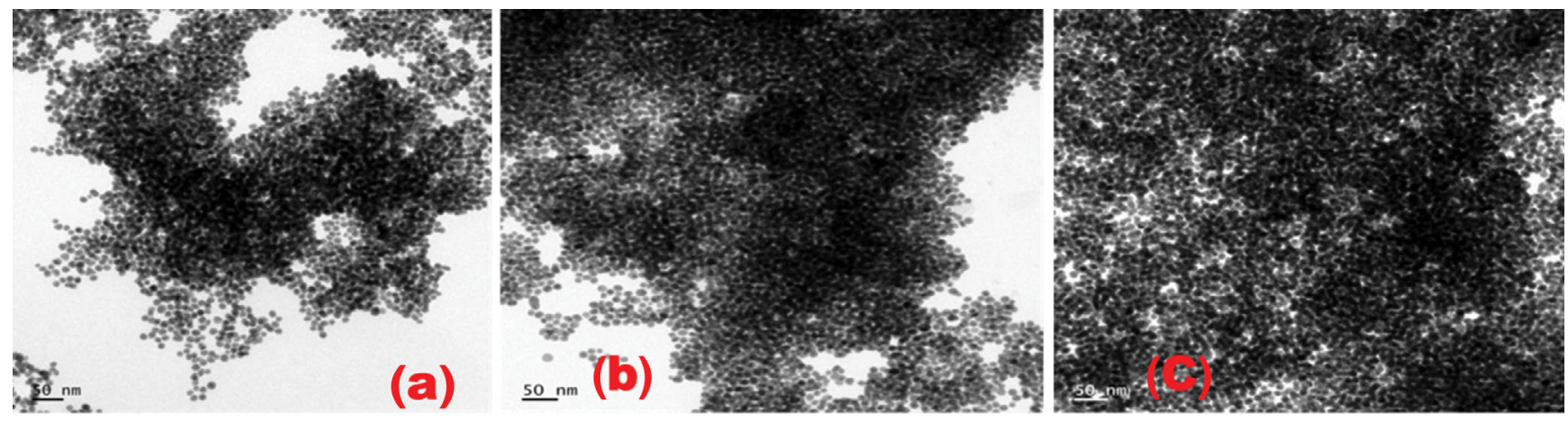

Fig. 7. TEM images of (a) $\beta$-CD-AuNP-S-HCQ (b) $\beta$-CD-AuNP-R-HCQ, and (c) $\beta$-CD-AuNP-RS-HCQ. 

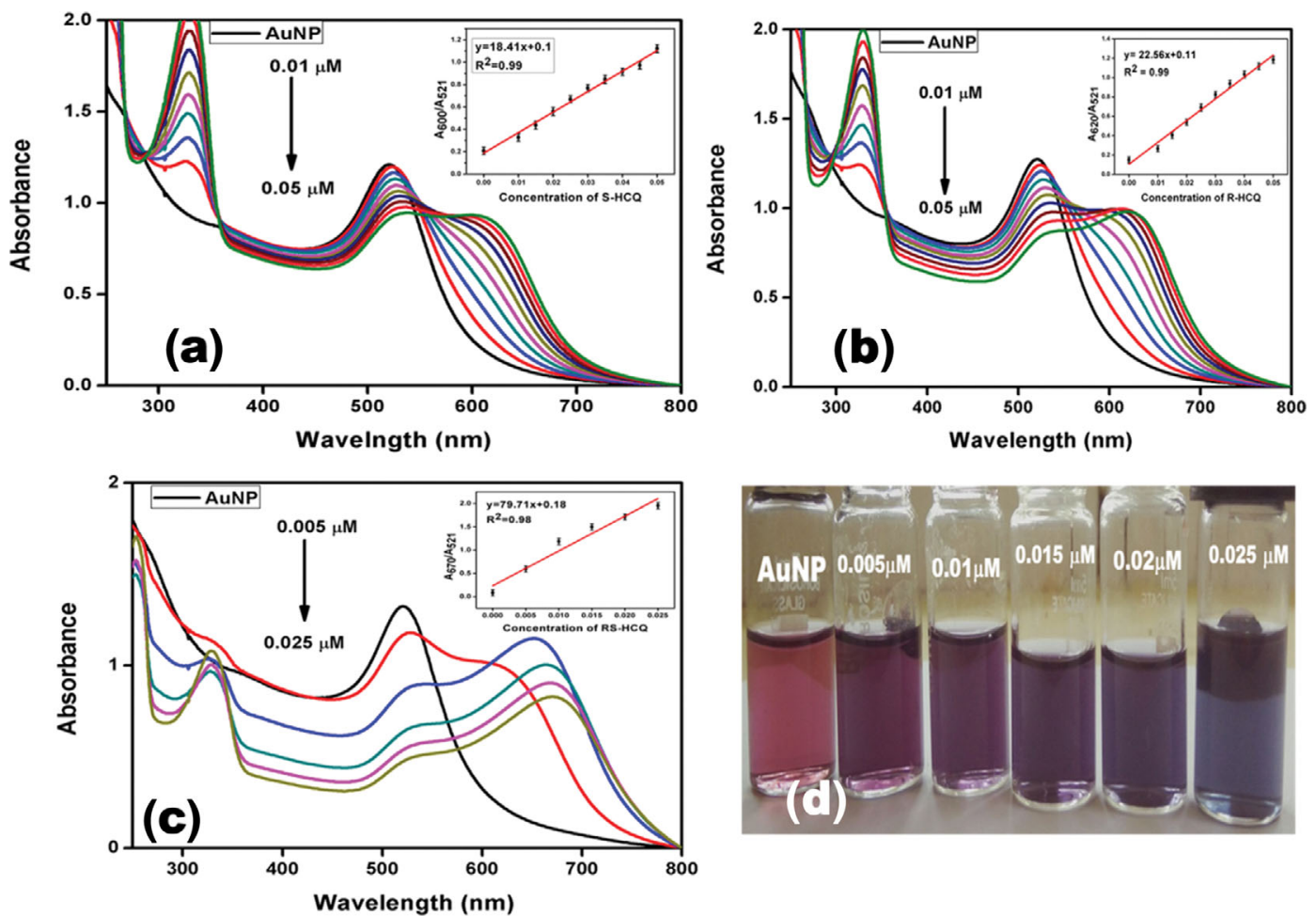

Fig. 8. UV-vis. spectra of different concentrations of (a) S-HCQ, (b) R-HCQ and (c) RS-HCQ in $\beta$-CD-AuNP. Inset: corresponding concentration versus $\mathbf{A} / \mathrm{A}_{521}$ graph. (d) Photographic image of $\beta$-CD-AuNP with increasing concentration of RS-HCQ.

ally decreased with the formation of new absorption peaks at 600 $\mathrm{nm}$ and $620 \mathrm{~nm}$ for SHCQ and RHCQ, respectively (Fig. 8(a) \& (b)). It is clear that the increase in aggregation generated by single enantiomeric drugs through electrostatic and hydrogen bonding is in the concentration range $0.01-0.05 \mu \mathrm{M}$. In the case of RS-HCQ, there is a fast decrease in the absorption peak at $521 \mathrm{~nm}$ and a new peak appears at $670 \mathrm{~nm}$ even at very low concentration $(0.005-$ $0.025 \mu \mathrm{M})$. This indicates the effectiveness in the binding of racemic drug with AuNP rather than the single enantiomer. According to this, the color changed from pink to purple (photographic image, Fig. 8(d)) and then to blue, which was consistent with spectroscopic data. The linear relationship between the concentration versus the ratio of $\mathrm{A}_{600} / \mathrm{A}_{521}, \mathrm{~A}_{620} / \mathrm{A}_{521}$ and $\mathrm{A}_{670} / \mathrm{A}_{521}$ plotted for $\beta$ CD-AuNP-S-HCQ, $\beta$-CD-AuNP-R-HCQ and $\beta$-CD-AuNP-RSHCQ is displayed in the inset of Fig. 8(a), (b) \& (c), respectively. The limit of detection (LOD) for $\beta$-CD-AuNP-SHCQ, $\beta$-CDAuNP-RHCQ and $\beta$-CD-AuNP-RS-HCQ was found to be 11.36 , 9.23 and $2.61 \mathrm{nM}$, respectively.

As the time increased the surface plasmon resonance of $\beta$-CDAuNP also varied in the presence of HCQ drugs. A strong shift in the absorption maximum occurred within 10 minutes on the addition of RS-HCQ, indicating the effective interaction of racemic drug with $\beta$-CD-AuNP (Fig. S3). This is attributed to the oligomers of $\alpha$-D-glucopyranose present in cyclodextrin which possess an intrinsic chirality that helps to form diastereomeric inclusion complexes with enantiomeric pairs [32,35]. Moreover, hydrogen bonding and electric dipole interaction between $\beta$-CD and HCQ also enhanced the sensing of enantiomeric HCQ.

\section{Selectivity Studies}

To validate the selectivity of the proposed sensing system, interfering and structurally similar compounds, including levofloxacin, quinine sulphate (QS), glucose, fructose, ascorbic acid, $\mathrm{Na}_{2} \mathrm{HPO}_{4}$, $\mathrm{NaNO}_{3}$ and $\mathrm{CaCl}_{2}$, were investigated under similar conditions. The
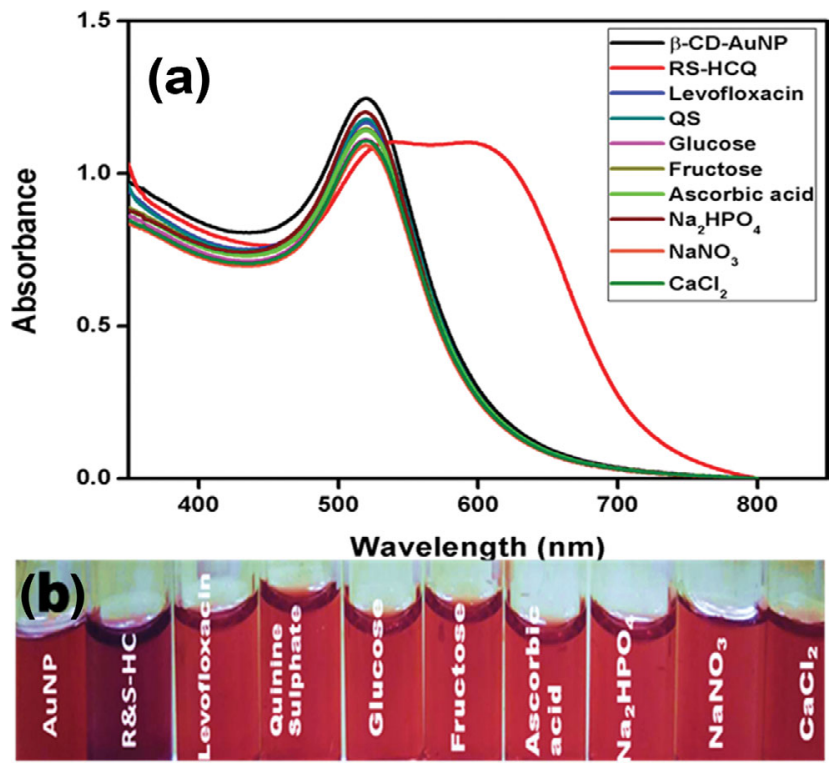

Fig. 9. UV-vis absorption spectra of (a) $\beta$-CD-AuNP on the addition of different interfering and structurally similar substance related to HCQ and (b) corresponding photographic image. 
UV-vis spectra of the selectivity studies and photographic image of the same are described in Fig. 9(a) \& (b). The above results suggest that the interfering substance has only negligible influence on the sensing of with $\beta$-CD-AuNP in the proposed system and high selectivity towards RS-HCQ. pH of the synthesized AuNP was found to be 10 under optimum condition. On the addition of S-HCQ, RHCQ and RS-HCQ the pH decreased to 8.2, 8.1 and 8, respectively. The decrease in the $\mathrm{pH}$ is due to the complexation of the enantiomeric drug molecule with $\beta$-CD-AuNP and that led to the red shift.

\section{Fluorescence Sensing of RS-HCQ Using $\beta$-CD-AuNP}

The fluorescence activity of the $\beta$-CD-AuNP and its interaction with RS-HCQ were determined by applying an excitation wavelength of $521 \mathrm{~nm}$. From Fig. 10(a), $\beta$-CD-AuNP exhibited a weak emission at $781 \mathrm{~nm}$. On the addition of RS-HCQ, an increase in the fluorescence peak was observed at $781 \mathrm{~nm}$. The addition of R-
HCQ also enhanced the fluorescence signal than that in the case of S-HCQ- $\beta$-CD-AuNP, but RS-HCQ showed higher intensity. This could be due to the intrinsic chirality of oligomers of $\alpha$-Dglucopyranose in the cyclodextrin which formed inclusion complexes with enantiomeric, resulting in the effective binding of RSHQC [32,35]. The effect of concentration of RS-HCQ was studied by monitoring its fluorescence spectra at different concentration (Fig. 10(b)). As the concentration of the drug increased, the fluorescence emission of each and racemic drug increased. The inset of Fig. 10(b) shows the linear relationship between the concentration with intensity, and the limit of detection was found to be $0.15 \mathrm{nM}$. The interaction of hydroxychloroquine drugs with AuNP induced the turn on effect on the fluorescence emission. Gold nanoclusters showed higher fluorescence emission than gold nanoparticles [39].
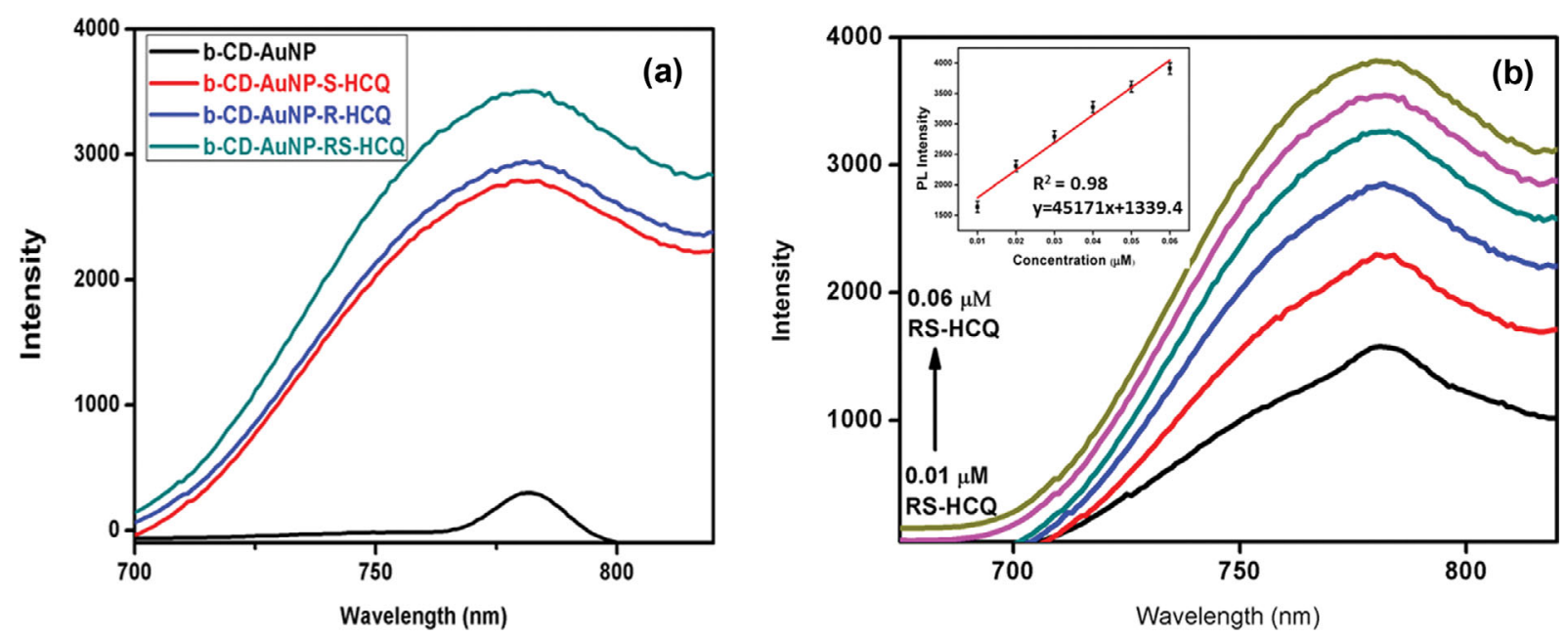

Fig. 10. Fluorescence spectra of (a) $\beta$-CD-AuNP with S-HCQ, R-HCQ and RS-HCQ and (b) different concentration of RS-HCQ with $\beta$-CDAuNP. Inset shows the linear plot of concentration with intensity.

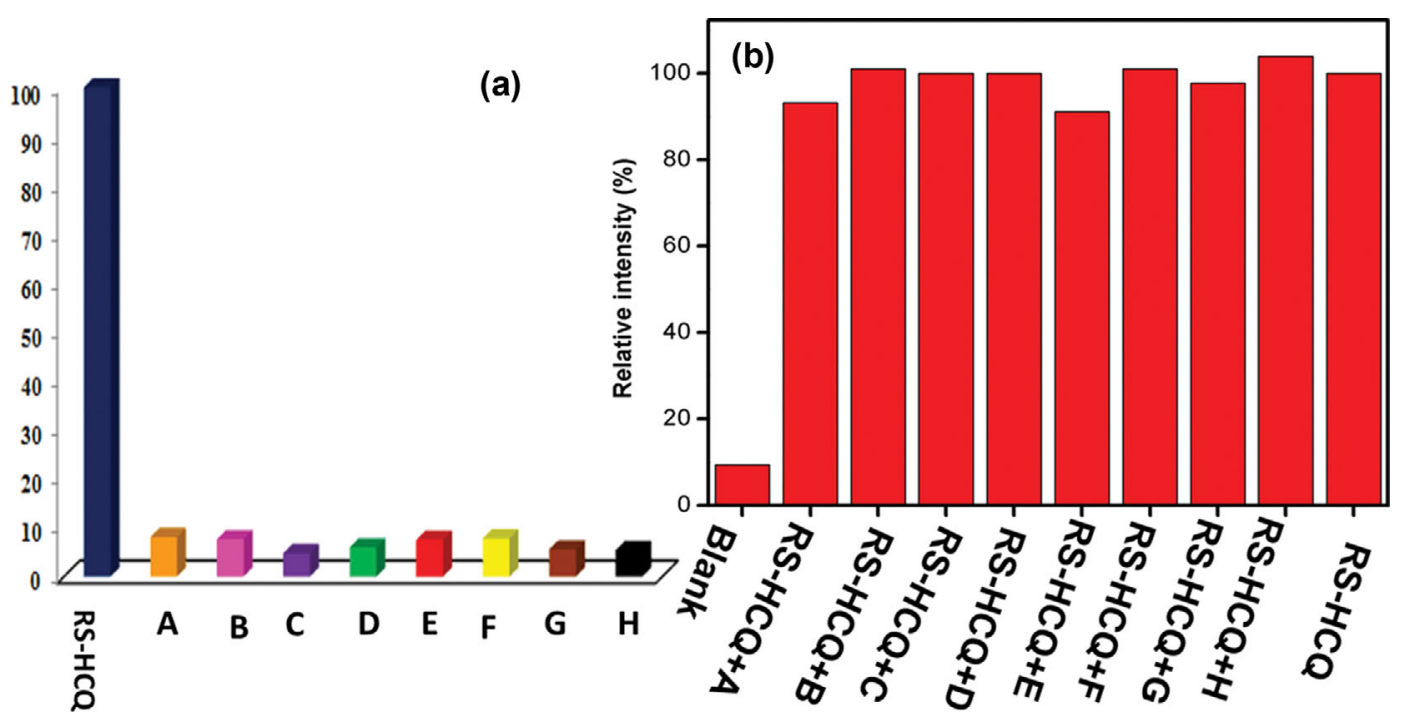

Fig. 11. Relative fluorescence intensity of $\beta$-CD-AuNP monitored at $820 \mathrm{~nm}$ in the presence of (a) RS-HCQ and various interfering and structurally similar compounds, (b) fluorescence intensity of interfering and various compounds with $\beta$-CD-AuNP-RS-HCQ. (A) levofloxacin, (B) quinine sulfate, (C) glucose, (D) fructose, (E) ascorbic acid, (F) $\mathrm{Na}_{2} \mathrm{HPO}_{4},(\mathrm{G}) \mathrm{NaNO}_{3}$ and $(\mathrm{H}) \mathrm{CaCl}_{2}$. 


\section{Selectivity and Interference Studies Towards RS-HCQ}

To confirm the sensitivity of the sensor towards RS-HCQ, various interfering and structurally similar compounds, including levofloxacin, quinine sulfate, glucose, fructose, ascorbic acid, $\mathrm{Na}_{2} \mathrm{HPO}_{4}$, $\mathrm{NaNO}_{3}$ and $\mathrm{CaCl}_{2}$ upon the addition with $\beta$-CD-AuNP were investigated. Fluorescence intensity at $820 \mathrm{~nm}$, measured after the addition of double the concentration of each substance, exhibited that a major fluorescence turn-on response solely occurred within the presence of RS-HCQ drug (Fig. 11(a)). This result demonstrated the high selectivity of the proposed sensing system, which is due to the highly specified interaction between $\beta$-CD-AuNP and RHCQ drugs. Fig. 11(b) also reveals the negligible interference occurring by other substances in the sensing of RS-HCQ, even with double the concentration of interfering substances.

\section{Electrochemical Sensing of RS-HCQ with $\beta$-CD-AuNP Modi-} fied AuE

The electrochemical behavior of RS-HCQ on bare AuE and $\beta$ $\mathrm{CD}$-AuNP/AuE was studied using cyclic voltammetry and differential pulse voltammetric technique in phosphate buffer solution (pH 6) containing $0.05 \mu \mathrm{M}$ RS-HCQ. The electrochemical procedure was undertaken by applying the potential in the range 0.6 to $1.3 \mathrm{~V}$ at a potential scan rate of $100 \mathrm{mV} / \mathrm{s}$. As shown in Fig. 12, bare AuE exhibited a weak oxidation signal, indicating slow electron transfer occurred in the electrode. After modification with $\beta$ CD-AuNP, the modified AuE showed higher oxidation current than bare GCE. This is due to the electrical conducting nature of gold nanoparticles incorporated in $\beta$-CD. In the presence of RSHCQ, the current increased significantly and also a negative shift in the potential describing the oxidation process occurred in the drug. The $\beta$-CD-AuNP/AuE was also used for the sensing RHCQ and SHCQ, and RHCQ showed higher current response than the SHCQ, but both are less than the current response obtained for $\beta$ $\mathrm{CD}-\mathrm{AuNP} / \mathrm{RS}-\mathrm{HCQ} / \mathrm{AuE}$. This is attributed to the distinctive sensing property of the $\beta$-CD-AuNP towards each drug molecule. Therefore, the electrochemical sensing studies also confirmed the interaction of $\beta$-CD-AuNP with HCQ drugs and the higher interaction of racemic HCQ.

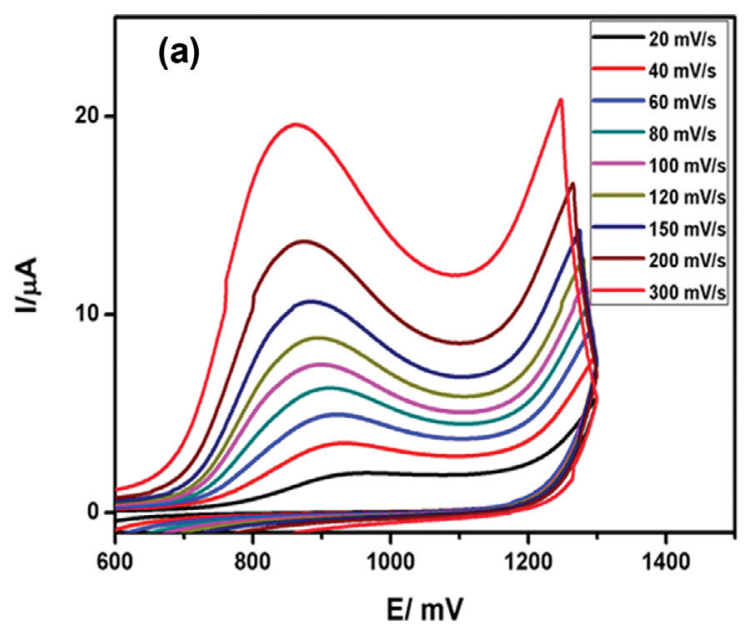

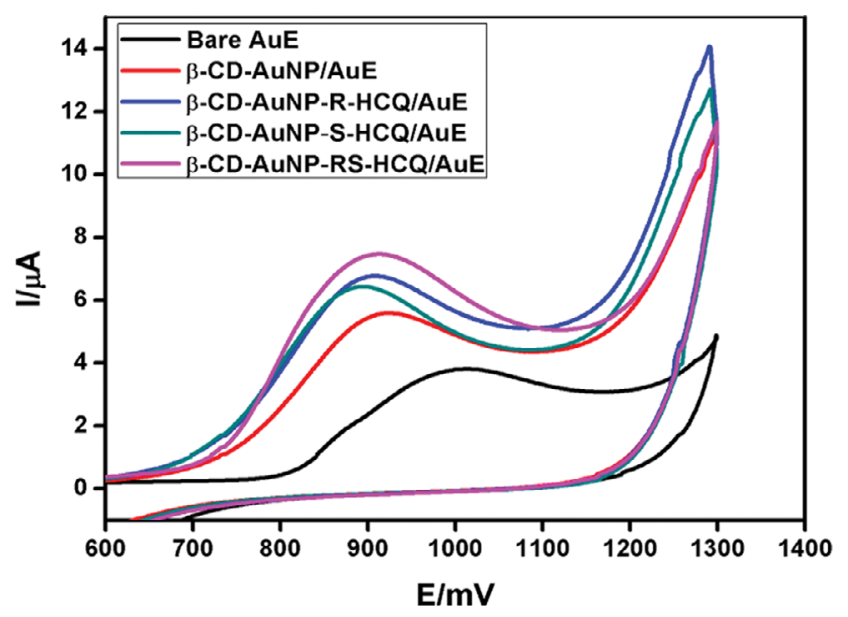

Fig. 12. CVs of bare AuE and $\beta$-CD-AuNP/AuE in the absence and presence of enantiomeric and single HCQ drug in $0.1 \mathrm{M}$ PBS ( $\mathrm{pH}=6)$ solution.

The number of electrons (n) for a completely irreversible process in the rate determining step can be calculated by using a simple formula:

$$
\alpha \mathrm{n}=\frac{47.7}{\mathrm{Ep}-\mathrm{Ep} / 2}
$$

where $\alpha$ is the electron transfer coefficient, Ep and Ep/2 represent the peak potential and the potential at which $\mathrm{I}=\mathrm{Ip} / 2$, respectively in the CV. The value for $\alpha$ n was found to be 0.41 . The number of electrons in the rate-determining step was $\mathrm{n}=1$, supposing the electron transfer coefficient was 0.369 [40] and the oxidation peak potential consistent with expectations for oxidation of a secondary or tertiary amine. The anodic peak in the CV may be due to the irreversible oxidation of the nitrogen of alkylamino side chain and the $\mathrm{N}$-heterocyclic nitrogen of the aminoquinoline moiety grouping of hydroxychloroquine molecule $[41,42]$.

\section{Effect of Scan Rate}

The effect of scan rate on the oxidation peak current was meas-

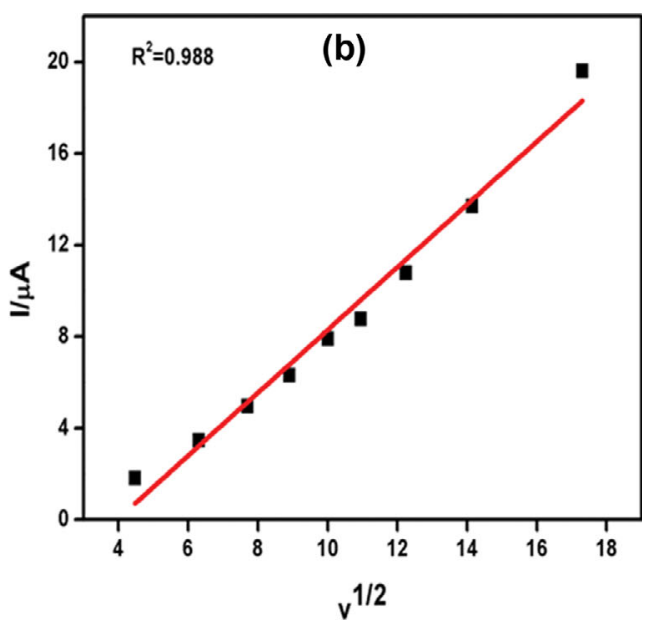

Fig. 13. (a) Cyclic voltammogram of $\beta$-CD-AuNP/AuE/RS-HCQ at different scan rates and (b) the linear plot of square root of scan rate versus peak current. 

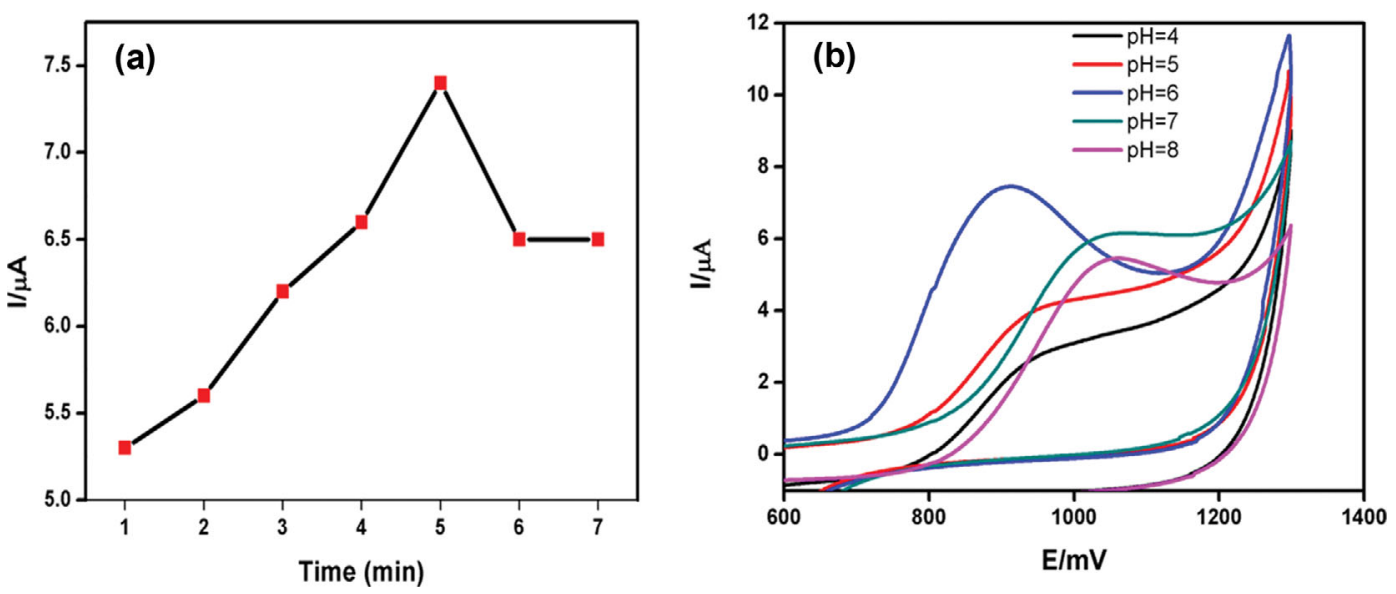

Fig. 14. (a) Effect of incubation time on the oxidation peak current of $\beta$-CD-AuNP/AuE and (b) effect of pH of PBS containing $0.05 \mu \mathrm{M}$ RSHCQ.

ured by changing the scan rate from $20-300 \mathrm{mV} / \mathrm{s}$. Fig. 13(a) depicts the increase of oxidation peak current with the increase of scan rate. A shift in the oxidation current to more positive values and the linearity observed in plot of I versus $\mathrm{v}^{1 / 2}$ (Fig. 13(b)), both suggest the diffusion controlled electrodic process [43].

\section{Effect of Incubation Time and pH}

Cyclic voltammetry studies were carried out to obtain the optimum $\mathrm{pH}$ and incubation time for the maximum current response of RS-HCQ sensing at the $\beta$-CD-AuNP/AuE. The incubation time was investigated to achieve maximum current response for RSHCQ at the $\beta$-CD-AuNP/AuE (Fig. 14(a)). As the incubation time increased, peak current also increased and reached maximum at 5 minutes. With further increase in time, the peak current reduced and remained constant. Thus, accumulation could enhance the sensitivity of determination and 5 minute incubation time was selected for further experimental studies. To investigate the optimum $\mathrm{pH}$ for the maximum response of the sensor, the $\mathrm{pH}$ of the buffer varied from 4 to 8 (Fig. 14(b)). A cathodic current gradually increased as the $\mathrm{pH}$ increased and reached a maximum at $\mathrm{pH}$

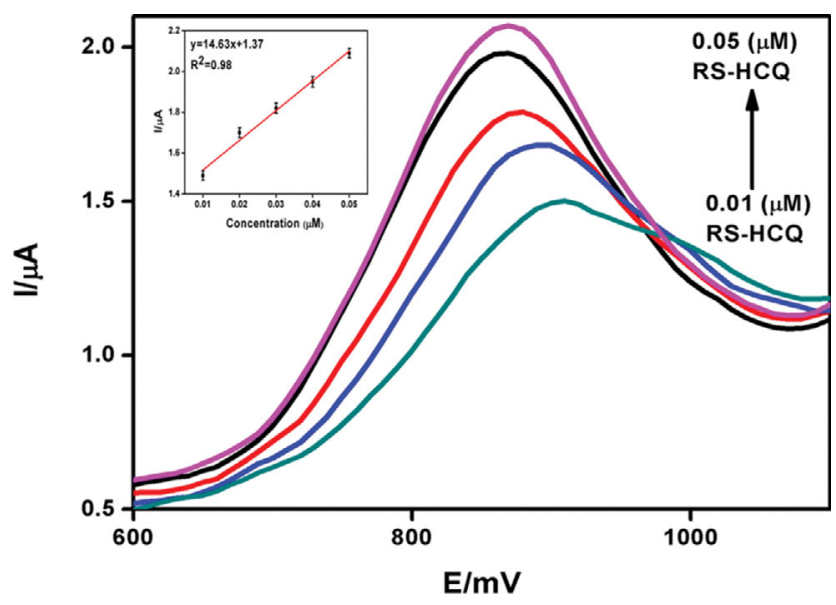

Fig. 15. DPVs of $\beta$-CD-AuNP/AuEelectrode at different concentrations of RS-HCQ ranging from 0.01 to $0.05 \mu \mathrm{M}$. Inset: linear plot of concentration versus peak current.
6 and then decreased with increase in $\mathrm{pH}$. The optimum $\mathrm{pH}$ selected for further studies was 6 . The proton concentration supported the electro-active oxidation of HCQ [41]. The shift in the cathodic peak current with increase in $\mathrm{pH}$ in the range between 4 and 7, suggests the effect of protonation in the electrodic process [43].

\section{Effect of Concentration}

Different concentrations of RS-HCQ in the range 0.01-0.05 $\mu \mathrm{M}$ were added into the electrochemical cell containing electrodes and buffer solution. The differential pulse voltammetric technique was used for the concentration studies with pulse width $200 \mathrm{~ms}$ and pulse amplitude $25 \mathrm{mV}$. The results obtained are plotted in Fig. 15. The inset in Fig. 15 shows the linear relationship between the peak current and concentration $\left(\mathrm{R}^{2}=0.985\right)$. The limit of detection for the sensing of RS-HCQ using $\beta$-CD-AuNP/AuE was found to be $0.85 \mathrm{nM}$. The newly constructed system exhibited higher sensitivity and better performance in the case of enantiomeric sensing of hydroxychloroquine drugs.

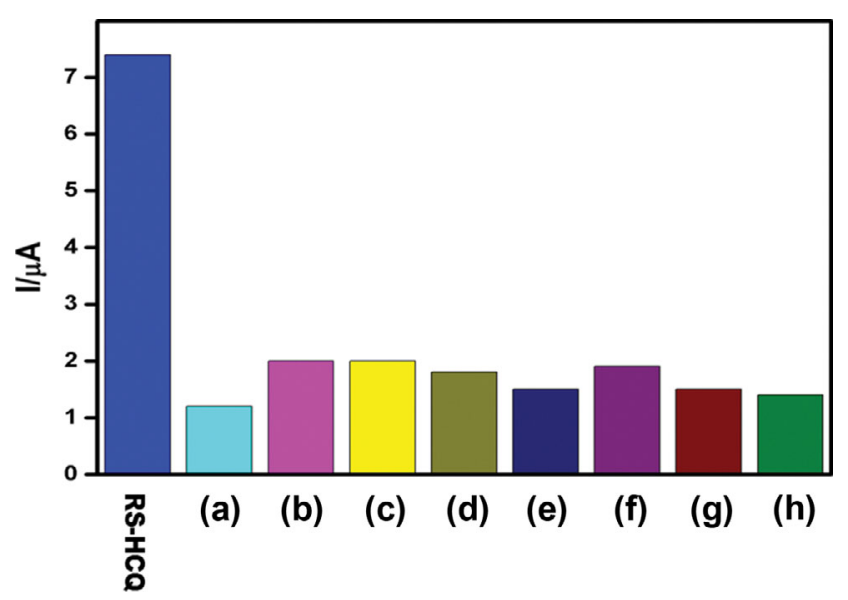

Fig. 16. Peak current from DPV measurements of $\beta$-CD-AuNP/AuE toward RS-HCQ and various interfering and structurally similar substances. (a) Levofloxacin, (b) quinine sulphate, (c) glucose, (d) fructose, (e) ascorbic acid, (f) $\mathrm{Na}_{2} \mathrm{HPO}_{4}$, (g) $\mathrm{NaNO}_{3}$ and (h) $\mathrm{CaCl}_{2}$. 


\section{Selectivity}

To investigate the selectivity of the developed system towards RS-HCQ, different interfering species was chosen and their electrochemical behavior studied using differential pulse voltammetric methods. The bar diagram shows (Fig. 16) the influence of some drugs, sugars, acids and some inorganic salts [44] on the RSHCQ sensor. Among the various interferences, significant current response was only with RS-HCQ. The low interference of ionic and non-ionic species is mainly due to the size, permeability and the electrostatic environment relative to RS-HCQ.

\section{Real Sample Analysis}

To illustrate the practical application in pharmaceutical analysis, optical, fluorescence and electrochemical methods were employed to determine hydroxychloroquine in its pharmaceutical samples. The standard addition method was carried out in which HCQS-200 (hydroxychloroquine $200 \mathrm{mg}$ ) was weighed and powdered using a mortar and pestle. Then this powder was dissolved in distilled water and filtered. From this solution different concentrations $(0.01-0.03 \mu \mathrm{M})$ were added into sample vials containing $2 \mathrm{~mL} \beta$-CD-AuNP, and the HCQ drug concentration was measured using the aforesaid procedure. The concentration of pharmaceutical drug sample was calculated by comparing the absorbance ratio obtained from standard RS-HCQ drugs. The photographic image of the RS-HCQ containing pharmaceutical sample solutions was obtained using $\beta$-CD-AuNP as a colorimetric probe (Fig. S4). The standard addition method was also used for the fluorescence spectroscopic investigation of real sample analysis. The electrochemical determination of HCQ in the pharmaceutical drug sample was also carried out using $\beta$-CD-AuNP/AuE using the standard addition method. The obtained results of real sample analysis are tabulated in Table S1.

Previous reports of the various methods for the detection HCQ drug and the LOD for the corresponding methods are tabulated in Table 2.

\section{CONCLUSION}

We have successfully developed fast and convenient microwave synthesised water dispersible AuNP using $\beta$-cyclodextrin as capping agent. $\beta$-CD support as a rational function of the surface of

Table 2. Comparison of various reported methods with for the sensing of $\mathrm{HCQ}$

\begin{tabular}{lcc}
\hline \hline \multicolumn{1}{c}{ Method/ Modified electrode } & LOD & References \\
\hline Spectrophotometry & $0.1 \mu \mathrm{M}$ & 26 \\
Differential pulse voltammetry (GCE) & $11.2 \mu \mathrm{M}$ & 25 \\
CPT-BDD electrode & $0.06 \mu \mathrm{M}$ & 25 \\
HCQ-PTA/CPE & $0.78 \mu \mathrm{M}$ & 27 \\
$\beta$-CD ionophore/CPE & $0.46 \mu \mathrm{M}$ & \\
GC/PAPA-SAM/GCE & $11.2 \mu \mathrm{M}$ & 45 \\
GC-PMPDA-SAM/GCE & $4.65 \mathrm{nM}$ & 46 \\
Optical & $2.61 \mathrm{nM}$ & This work \\
Fluorescence & $0.15 \mathrm{nM}$ & This work \\
Electrochemical & $0.85 \mathrm{nM}$ & This work \\
\hline
\end{tabular}

AuNP provides the strength of suitable interactions (hydrogen bonding and electrostatic) with RS-HCQ and its enantiomeric drugs. $\beta$-CD-AuNP acts as optical, fluorescence and electrochemical sensing probe for the detection of RS-HCQ. The color change from pink to blue indicates the addition of HCQ to $\beta$-CD-AuNP. Various characterization techniques confirmed the induced aggregation between $\beta$-CD-AuNP and HCQ through hydrogen bonding and electrostatic interaction. The dipole moment variation in enantiomers of HCQ and intrinsic chirality of $\beta$-CD also supports the different interactions between RS-HCQ and enantiomers of HCQ. The limit of detection was 2.61, $0.15,0.85 \mathrm{nM}$ for optical, fluorescence and electrochemical methods, respectively, and the successful determination of HCQ drug in pharmaceutical samples is also possible. Thus, the fabricated $\beta$-CD-AuNP probe paves the way to a new, successful analysis platform for the sensing of enantiomeric hydroxychloroquine drug with high selectivity and sensitivity.

\section{ACKNOWLEDGEMENTS}

JMG gratefully acknowledges University Grants Commission (UGC), for the award of research fellowship, and Department of Science \& Technology (DST), Government of India for instrumentation facilities by DST-PURSE Phase II.

\section{CONFLICT OF INTEREST}

We have no conflict of interest to declare.

\section{SUPPORTING INFORMATION}

Additional information as noted in the text. This information is available via the Internet at http://www.springer.com/chemistry/ journal/11814.

\section{REFERENCES}

1. A. Aravind, M. Sebastian and B. Mathew, New J. Chem., 42, 1502 (2018).

2. S. K. Kailasa, V. N. Mehta and H. F. Wu, RSC Adv., 4, 16188 (2014).

3. M. Sebastian, A. Aravind and B. Mathew, Mater. Res. Express, 5, 085015 (2018).

4. D. Vilela, M. C. González and A. Escarpa, Anal. Chim. Acta, 751, 24 (2012).

5. K. Saha, S. S. Agasti, C. Kim, X. Li and V. M. Rotello, Chem. Rev., 112, 2739 (2012).

6. J. Khodaveisi, S. Dadfarnia, A. M. H. Shabani and D. Saberi, Sens. Actuators B Chem., 239, 1300 (2017).

7. J. Kang, Y. Zhang, X. Li, L. Miao and A. Wu, ACS Appl. Mater. Interfaces, 8, 1 (2015).

8. C. Fang, R. Dharmarajan, M. Megharaj and R. Naidu, TrAC-Trends Anal. Chem., 86, 143 (2017).

9. M. Hu, J. Chen, Z.-Y. Li, L. Au, G. V. Hartland, X. Li, M. Marquez and Y. Xia, Chem. Soc. Rev., 35, 1084 (2006).

10. M.-C. Daniel and D. Astruc, Chem. Rev., 104, 293 (2004).

11. C. Fan, S. Wang, J. W. Hong, G. C. Bazan, K. W. Plaxco and A. J. 
Heeger, Proc. Natl. Acad. Sci. USA, 100, 6297 (2003).

12. J. Pikkel, O. Chassid, A. Sharabi-Nov and I. Beiran, Graefes Arch. Clin. Exp. Ophthalmol., 251, 1687 (2013).

13. C. R. Stelton, D. B. Connors, S. S. Walia and H. S. Walia, Clin. Rheumatol., 32, 895 (2013).

14. J. J. Chen, R. M. Tarantola, C. N. Kay and V. B. Mahajan, Eye Rounds. Org. (2011).

15. E. B. Gouveia, M.S. de Á. Morales, G. B. Gouveia and V.P.M. Lourenzi, Arq. Bras. Oftalmol., 70, 1046 (2007).

16. R. V. Shearer and E. L. Dubois, Am. J. Ophthalmol., 64, 245 (1967).

17. A. K. Singh, A. Singh, A. Shaikh, R. Singh and A. Misra, Diabetes Metab. Syndr., 14, 241 (2020).

18. L. A. Percy and M. A. Fang, Rheum. Dis. Clin. North Am., 26, 433 (2000).

19. D. R. Brocks, F. M. Pasutto and F. Jamali, J. Chromatogr. B, 581, 83 (1992).

20. P. Volin, J. Chromatogr. B, 666, 347 (1995).

21. Y. Wei, G. A. Nygard and S. K. W. Khalil, J. Liq. Chromatogr., 17, 3479 (1994).

22. S. B. Williams, L. C. Patchen and F.C. Churchill, J. Chromatogr., 433, 197 (1988).

23. R. G. Morris, J. Chromatogr. B, 338, 422 (1985).

24. L. Z. Wang, R. Y. L. Ong, T. M. Chin, W. L. Thuya, S. C. Wan, A. L. A. Wong, S.-Y. Chan, P. C. Ho and B. C. Goh, J. Pharm. Biomed. Anal., 61, 86 (2012).

25. P. B. Deroco, F. C. Vicentini, G. G. Oliveira, R. C. Rocha-Filho and O. Fatibello-Filho, J. Electroanal. Chem., 719, 19 (2014).

26. M. L. P. M. Arguelho, J.F. Andrade and N. R. Stradiotto, J. Pharm. Biomed. Anal., 32, 269 (2003).

27. M. M. Khalil, G. M. A. El-aziz and A. Ashry, J. Iran. Chem. Soc., 15, 2411 (2018).

28. G. Crini, Chem. Rev., 114, 10940 (2014).
29. N. Sharma and A. Baldi, Drug Deliv., 23, 739 (2016).

30. K. A. Connors, Chem. Rev., 97, 1325 (1997).

31. L. Liu and Q.X. Guo, J. Inclusion Phenom. Macrocyclic Chem., 42, 1 (2002).

32. S. D. Chatziefthimiou, M. Inclán, P. Giastas, A. Papakyriakou, K. Yannakopoulou and I. M. Mavridis, Beilstein J. Org. Chem., 13, 1572 (2017).

33. A. Shpigun, I. A. Ananieva, N. Y. Budanova and E. N. Shapovalova, Russ. Chem. Rev., 72, 1035 (2003).

34. S. Joseph and B. Mathew, Spectrochim. Acta, Part A, 136, 1371 (2015).

35. T. Huang, F. Meng and L. Qi, J. Phys. Chem. C, 113, 13636 (2009).

36. R. Vijayan, S. Joseph and B. Mathew, Artif. Cell Nanomed. B, 46, 861 (2017).

37. J. M. George, R. N. Priyanka and B. Mathew, Microchem. J., 155, 104686 (2020).

38. R. Vijayan, S. Joseph and B. Mathew, IET Nanobiotechnol., 12, 850 (2018).

39. T. N. Nguyen, T. H. Do, D. H. Nguyen, D. Vu, Q. H. Do, H. N. Tran and T. H. L. Nghiem, Appl. Phys. Express, 9, 022001 (2016).

40. J. M. George and B. Mathew, J. Iran. Chem. Soc., 17, 2613 (2020).

41. K. Lam, S. J. Van Wyck and W. E. Geiger, J. Electroanal. Chem., 799, 531 (2017).

42. M. Srivastava, P. Tiwari, V. K. Mall, S. K. Srivastava and R. Prakash, Microchim. Acta, 186, 415 (2019).

43. M. L. Arguelho, J. F. Andrade and N. R. Stradiotto, J. Pharm. Biomed. Anal., 32, 269 (2003).

44. R. I. Fox, Semin Arthritis Rheum., 2, 82 (1993).

45. A. Khoobi, S. M. Ghoreishi and M. Behpour, Analyst, 139, 4064 (2014).

46. A. Khoobi, S. M. Ghoreishi, M. Behpour, M. Shaterian and M. Salavati-Niasari, Colloids Surf. B, 123, 648 (2014). 